\title{
Editorial Introduction to the Special Issue from the International Symposium on Biomarkers for Alzheimer's Disease and Related Disorders
}

Marwan N. Sabbagh

\section{INTRODUCTION}

Alzheimer's disease (AD) is a long and continuous neurodegenerative condition that starts with demonstrable pathologic changes decades before the onset of symptoms and ends with symptomatic dementia [1, 2]. There have been significant advancements in the field of biomarker-driven diagnostics, including the development of amyloid positron emission tomography [carbon 11-labeled Pittsburgh Compound B (C-11 PiB)] [3, 4] and the approvals of the fluorine 18 derivatives florbetapir [5], florbetaben [6], and flutemetamol [7], as well as advancements in cerebrospinal fluid (CSF) testing [8]. Nonetheless, no consensus has been reached regarding the routine incorporation of biomarkers into clinical evaluation; various groups have issued differing recommendations [9-11]. What is missing from the clinical environment is a widely accepted, reproducible, and valid peripheral (e.g., blood, urine, or saliva) biomarker, much like the prostate-specific antigen screen for prostate cancer or the glycosylated hemoglobin $\left(\mathrm{HbA}_{1 \mathrm{c}}\right)$ measure for diabetes mellitus. Plasma amyloid has been measured extensively [12]. Recent reports suggest that

M. N. Sabbagh $(\bowtie)$

Department of Neurology, Barrow Neurological Institute, St. Joseph's Hospital and Medical Center, Phoenix, AZ, USA

e-mail: Neuropub@barrowneuro.org measuring plasma tau might be desirable and could potentially have validity as a peripheral diagnostic biomarker [13].

The first International Symposium on Biomarkers for Alzheimer's Disease and Related Disorders was held on 26 October 2016 at the National Taiwan University Hospital International Convention Center. It was organized by the Department of Neurology, National Taiwan University Hospital, and co-organized by the Taiwan Dementia Society and the Molecular Imaging Center at National Taiwan University. In this international symposium, global and regional experts gathered to consider the state of diagnostics for Alzheimer's dementia and to discuss the advancement of peripheral diagnostics for detection of and diagnosis of AD.

Dr. Beach's [14] review, "A Review of Biomarkers for Neurodegenerative Disease: Will They Swing Us Across the Valley?", points out critical flaws in clinical trial methodology and clinical diagnosis. Clinical diagnosis is inaccurate, and clinical trials in $\mathrm{AD}$ use cognitive end points that lack disease specificity. His review points out the gap between the promises of basic science discoveries and the failures of clinical trials of novel drugs because of the imprecision of clinical end points. His argument is to incorporate biomarker-driven outcomes, which has been done successfully in cancer trials and might require the use of multiple biomarkers. 
Dr. Blennow's [15] report, "A Review of Fluid Biomarkers for Alzheimer's Disease-Moving from CSF to Blood", reviews the well-recognized CSF biomarkers of amyloid $\beta 42$ (Aß42), tau, and p-tau and comments about the emergence of CSF neurogranin as a predictor of cognitive decline in AD. He also describes how advancements in laboratory techniques have enabled the detection of brain-specific tau and neurofilament light in plasma and how they might predict clinical progression. He further posits that such assays might be useful as a screening tool for $\mathrm{AD}$ that could be deployed in primary care.

Dr. Lue et al.'s [16] submission, "Amyloid Beta and Tau as Alzheimer's Disease Blood Biomarkers: Promise from New Technologies", points out that levels of plasma $A \beta$ and tau are much lower than CSF levels and that there are many proteins in blood that interfere with multiplex assays, making levels of AD-specific markers difficult to detect, track, and interpret. The review introduces the novel assay detection methods immunomagnetic reduction (IMR) and single molecule array (SIMOA), both of which have the ability to measure plasma tau. Quantification with both platforms is based on immunoreactivity between specific antibodies and analytes or protein standards. However, the principles and designs of the detection systems differ. IMR technology detects alternating-current magnetic susceptibility through the use of a superconducting quantum interference device (SQUID), while SIMOA technology detects the presence of antigens by fluorescence imaging of single enzyme-labeled immunocomplexes reacting with the fluorogenic substrate resorufin $\beta$-D-galactopyranoside (RGP) [16]. These technologies permit detection of $\mathrm{AD}$-specific markers in the periphery, which could be most useful in the development of peripheral biomarkers.

The introduction of IMR technology by Dr. Lue is followed by "Detection of Plasma Biomarkers Using Immunomagnetic Reduction: A Promising Method for the Early Diagnosis of Alzheimer's Disease", by Dr. Yang and colleagues [17], which provides technical details about how the IMR assay works, including an illustration of the designs and the preparations of the reagents and the IMR analyzer used for assaying $A \beta$ and tau protein. Dr. Yang et al. demonstrate that IMR, compared with the enzyme-linked immunosorbent assay (ELISA), has a 2-3 times lower limit of detection than the ELISA for assaying A $\beta$ and tau protein. The case is made for the feasibility of using IMR to assay plasma $A \beta$ and tau as a potential diagnostic test, with clinical sensitivity and specificity higher than $80 \%$ for discriminating patients with $\mathrm{AD}$ from patients without $\mathrm{AD}$ and from patients with mild cognitive impairment.

In Dr. Jhou and Dr. Tai's [18] review, "The Study of Postmortem Human Synaptosomes for Understanding Alzheimer's Disease and Other Neurological Disorders: A Review", they examine the literature related to synaptosomes and its relevance to AD research. Synaptic dysfunction is thought to play an important role in the pathophysiology of many neurologic diseases, including $\mathrm{AD}$, and previous studies have shown that synapse loss correlates with cognitive decline in AD. Synaptosomes are detached and resealed synaptic terminals; by examining them, researchers can gain insights into interneuronal interactions. This review summarizes the methods and the novel molecular analysis techniques by which synaptosomes are isolated from banked human brain tissue, such as RNA deep sequencing, shotgun proteomics, metabolomics, flow cytometry, and super-resolution imaging.

One particular patient group for whom detection of plasma tau or isolation of synaptosomes might be consequential is patients with Down syndrome (DS). In "A Review of Biomarkers for Alzheimer's Disease in Down Syndrome", Dr. Lee and colleagues [19] summarize a rapidly growing area of research, the convergence of approaches to $\mathrm{AD}$ and $\mathrm{DS}$ research. Dementia in DS is recognized as a common presenile dementia because of the overexpression of amyloid precursor protein in trisomy 21. The initial presentation of AD in DS may be difficult to recognize because of underlying intellectual disabilities. Use of biomarkers may be beneficial as a tool for detecting people with DS who are at risk of developing AD. Baseline plasma $A \beta$ and tau levels are elevated in patients with DS compared to controls. In addition, other markers related to telomere length, neuroinflammation, and methylation have been correlated with AD progression [19]. 
The contribution, "Increasing Precision of Clinical Diagnosis of AD Using a Combined Algorithm Incorporating Clinical and Novel Biomarker Data" [20], by Mannagh et al. discusses how $\mathrm{AD}$ diagnosis continues to be a diagnosis of exclusion despite the availability of advanced biomarker-driven diagnostic modalities. Because physicians often do not feel comfortable making a diagnosis of $\mathrm{AD}$, we propose an updated approach for detecting and diagnosing $\mathrm{AD}$ to improve physicians' diagnostic confidence. Our approach is clinically oriented, with structured interviews, aggregate risk analysis, bedside testing, and the use of plasma tau (IMR) and apolipoprotein E. The rationale and justification for each step are included.

\section{ACKNOWLEDGEMENTS}

This work was supported by NIA P30 AG019610, the Arizona Alzheimer's Research Consortium, and the Barrow Neurological Foundation. No funding or sponsorship was received for the publication of this article. The named author meets the International Committee of Medical Journal Editors (ICMJE) criteria for authorship for this manuscript, takes responsibility for the integrity of the work as a whole, and has given final approval for the version to be published. The author thanks the staff of Neuroscience Publications at Barrow Neurological Institute for assistance with manuscript preparation.

Disclosures. Dr. Marwan N. Sabbagh holds stock or has ownership in Muses Labs, Inc.; Versanum, Inc.; and Brain Health, Inc. He serves in an advisory capacity for Biogen; Eli Lilly and Co.; and vTv Therapeutics, Inc. He is a Research Investigator for studies supported by $\mathrm{AC}$ Immune; Eli Lilly and Co.; Biogen; Merck \& Co., Inc.; vTv Therapeutics, Inc.; F. Hoffmann-La Roche Ltd.; Lundbeck; Avid Radiopharmaceuticals; and Axovant Sciences, Inc.

Compliance with Ethics Guidelines. This article is based on previously conducted studies and does not involve any new studies of human or animal subjects performed by any of the authors.

Open Access. This article is distributed under the terms of the Creative Commons Attribution-NonCommercial 4.0 International License (http://creativecommons.org/licenses/ by-nc/4.0/), which permits any noncommercial use, distribution, and reproduction in any medium, provided you give appropriate credit to the original author(s) and the source, provide a link to the Creative Commons license, and indicate if changes were made.

\section{REFERENCES}

1. Jack CR Jr, Knopman DS, Jagust WJ, et al. Hypothetical model of dynamic biomarkers of the Alzheimer's pathological cascade. Lancet Neurol. 2010;9(1):119-28 (epub 2010/01/20).

2. Bateman RJ, Xiong C, Benzinger TL, et al. Clinical and biomarker changes in dominantly inherited Alzheimer's disease. N Engl J Med. 2012;367(9):795-804 (epub 2012/07/13).

3. Klunk WE, Engler $\mathrm{H}$, Nordberg $\mathrm{A}$, et al. Imaging brain amyloid in Alzheimer's disease with Pittsburgh Compound-B. Ann Neurol. 2004;55(3):306-19 (epub 2004/03/03).

4. Mathis CA, Mason NS, Lopresti BJ, Klunk WE. Development of positron emission tomography beta-amyloid plaque imaging agents. Semin Nucl Med. 2012;42(6):423-32 (epub 2012/10/03).

5. Clark CM, Schneider JA, Bedell BJ, et al. Use of florbetapir-PET for imaging $\beta$-amyloid pathology. JAMA. 2011;305(3):275-83 (epub 2011/01/20).

6. Sabri O, Sabbagh MN, Seibyl J, et al. Florbetaben PET imaging to detect amyloid beta plaques in Alzheimer's disease: phase 3 study. Alzheimers Dementia. 2015;11(8):964-74 (epub 2015/04/01).

7. Curtis C, Gamez JE, Singh U, et al. Phase 3 trial of flutemetamol labeled with radioactive fluorine 18 imaging and neuritic plaque density. JAMA Neurol. 2015;72(3):287-94 (epub 2015/01/27).

8. Shaw LM, Vanderstichele H, Knapik-Czajka M, et al. Cerebrospinal fluid biomarker signature in Alzheimer's disease neuroimaging initiative subjects. Ann Neurol. 2009;65(4):403-13 (epub 2009/03/20). 
9. McKhann GM, Knopman DS, Chertkow H, et al. The diagnosis of dementia due to Alzheimer's disease: recommendations from the National Institute on Aging-Alzheimer's Association workgroups on diagnostic guidelines for Alzheimer's disease. Alzheimers Dementia. 2011;7(3):263-9 (epub 2011/04/26).

10. Albert MS, DeKosky ST, Dickson D, et al. The diagnosis of mild cognitive impairment due to Alzheimer's disease: recommendations from the National Institute on Aging-Alzheimer's Association workgroups on diagnostic guidelines for Alzheimer's disease. Alzheimers Dementia. 2011;7(3):270-9 (epub 2011/04/26).

11. Dubois B, Feldman HH, Jacova C, et al. Advancing research diagnostic criteria for Alzheimer's disease: the IWG-2 criteria. Lancet Neurol. 2014;13(6):614-29 (epub 2014/05/23).

12. Figurski MJ, Waligorska T, Toledo J, et al. Improved protocol for measurement of plasma $\beta$-amyloid in longitudinal evaluation of Alzheimer's Disease Neuroimaging Initiative study patients. Alzheimers Dementia. 2012;8(4):250-60 (epub 2012/07/04).

13. Mattsson N, Zetterberg H, Janelidze S, et al. Plasma tau in Alzheimer disease. Neurology. 2016; 87(17):1827-35 (epub 2016/10/26).

14. Beach TG. A review of biomarkers for neurodegenerative disease: will they swing us across the valley? Neurol Ther. 2017. doi:10.1007/s40120-017-0072-x.
15. Blennow K. A review of fluid biomarkers for Alzheimer's disease-moving from CSF to blood. Neurol Ther. 2017. doi:10.1007/s40120-017-0073-9.

16. Lue LF, Guerra A, Walker DG. Amyloid beta and tau as Alzheimer's disease blood biomarkers: promise from new technologies. Neurol Ther. 2017. doi:10. 1007/s40120-017-0074-8.

17. Yang S-Y, Chiu MJ, Chen T-F, et al. Detection of plasma biomarkers using immunomagnetic reduction: a promising method for the early diagnosis of Alzheimer's disease. Neurol Ther. 2017. doi:10. 1007/s40120-017-0075-7.

18. Jhou J-F, Tai H-C. The study of postmortem human synaptosomes for understanding Alzheimer's disease and other neurological disorders: a review. Neurol Ther. 2017. doi:10.1007/s40120-017-0070-z.

19. Lee N-C, Chien Y-H, Hwu W-L. A review of Biomarkers for Alzheimer's disease in Down syndrome. Neurol Ther. 2017. doi:10.1007/s40120017-0071-y.

20. Sabbagh MN, Lue L-F, Fayard D, et al. Increasing precision of clinical diagnosis of AD using a combined algorithm incorporating clinical and novel biomarker data. Neurol Ther. 2017. doi:10.1007/ s40120-017-0069-5. 